\title{
Proof of the quantitative potential of immunofluorescence by mass spectrometry
}

\author{
Maria I Toki ${ }^{1}$, Fabiola Cecchi ${ }^{2}$, Todd Hembrough ${ }^{2}$, Konstantinos N Syrigos ${ }^{3}$ and David L Rimm ${ }^{1,4}$
}

Protein expression in formalin-fixed, paraffin-embedded patient tissue is routinely measured by Immunohistochemistry (IHC). However, IHC has been shown to be subject to variability in sensitivity, specificity and reproducibility, and is generally, at best, considered semi-quantitative. Mass spectrometry (MS) is considered by many to be the criterion standard for protein measurement, offering high sensitivity, specificity, and objective molecular quantification. Here, we seek to show that quantitative immunofluorescence (QIF) with standardization can achieve quantitative results comparable to MS. Epidermal growth factor receptor (EGFR) was measured by quantitative immunofluorescence in 15 cell lines with a wide range of EGFR expression, using different primary antibody concentrations, including the optimal signalto-noise concentration after quantitative titration. QIF target measurement was then compared to the absolute EGFR concentration measured by Liquid Tissue-selected reaction monitoring mass spectrometry. The best agreement between the two assays was found when the EGFR primary antibody was used at the optimal signal-to-noise concentration, revealing a strong linear regression $\left(R^{2}=0.88\right)$. This demonstrates that quantitative optimization of titration by calculation of signal-to-noise ratio allows QIF to be standardized to MS and can therefore be used to assess absolute protein concentration in a linear and reproducible manner.

Laboratory Investigation (2017) 97, 329-334; doi:10.1038/labinvest.2016.148; published online 16 January 2017

Immunohistochemistry (IHC) analysis of formalin-fixed, paraffin-embedded (FFPE) patient tissue is routinely used to measure common biomarker expression in diagnostic practice. Despite its widespread use, IHC is subject to variability in sensitivity, specificity and reproducibility, and is generally, at best, considered semi-quantitative. ${ }^{1,2}$ The scoring systems most commonly used, either ordinal or nominal, generate discontinuous, highly subjective data. As such IHC, and protein measurement in general has been considered unreliable as a companion diagnostic test. DNA-based testing, including in situ hybridization for amplification and translocations, and DNA sequencing, has supplanted protein assessment in the companion diagnostic space. Sometimes there is no nucleic acid-based method that can determine the best drug for a given patient and protein measurement would represent a better option, if it were as quantitative and objective as DNA testing.

Toward that goal, we have sought to define methods that can allow protein-based testing to be as quantitative and reproducible as nucleic acid testing. One such method is the AQUA method of automated quantitative immunofluorescence $(\mathrm{QIF})^{3}$ which calculates protein expression on a continuous scale by quantifying immunofluorescence pixel intensity per unit area. As such, it has been proven as a tool for removing the subjectivity of the traditional scoring system and providing objective and reproducible measurement of targets. However, immunofluorescence (IF) is still considered by some to be only semi-quantitative due to the nature of the detection and measurement methods, which often include an enzymatic amplification of the visualization reagent. When measuring protein, mass spectrometry (MS) is broadly recognized as among the most accurate of methods. It offers high sensitivity, specificity and objective molecular quantification ${ }^{4-7}$ of proteins in biological samples and, when coupled with selected reaction monitoring (SRM) methods, it can represent a criterion standard for protein measurement. Specifically, the development of mass spectrometry-based liquid tissue-selected reaction monitoring (LT-SRM) assay, enables the quantification of multiple proteins directly in FFPE patient tumor tissue in a linear fashion across a large range of concentrations, ${ }^{8,9}$ obviating the need for antibodies or other types of affinity reagents. The LT-SRM assay has been demonstrated to

\footnotetext{
${ }^{1}$ Department of Pathology, Yale University Medical School, New Haven, CT, USA; ${ }^{2}$ Nantomics Inc., Rockville, MD, USA; ${ }^{3} 3$ rd Department of Medicine, University of Athens, School of Medicine, Sotiria General Hospital, Athens, Greece and ${ }^{4}$ Department of Internal Medicine, Yale University Medical School, New Haven, CT, USA

Correspondence: Dr DL Rimm, MD, PhD, Professor of Pathology, Director, Yale Pathology Tissue Services, Department of Pathology, BML 116, Yale University School of Medicine, 310 Cedar St. PO Box 208023, New Haven 06520-8023, CT, USA.

E-mail: david.rimm@yale.edu

Received 6 November 2016; revised 4 December 2016; accepted 5 December 2016
} 
accurately measure expression of Epidermal Growth Factor Receptor (EGFR) in patient tumors. ${ }^{10}$ Clinical studies are currently defining quantitative cutoffs for EGFR expression that correlate to patient outcomes in various indications. The assay is run in CAP-certified CLIA laboratory and is currently used by physicians for clinical decision making. Here, we determine whether IF can be standardized to mass spectrometry to allow accurate and linear measurement of EGFR as a proof of concept that QIF can be performed as a quantitative method.

\section{MATERIALS AND METHODS Cell lines}

The cell lines MCF7, HT29, SKBR3, H441, H1355, H1993, H1648, A549, A431, H820, PC9, HCC193, H1975, H2882, and H1650 were purchased from the American Type Culture Collection (Manassas, VA, USA) or donated by other labs. Although the cell lines were not authenticated by our laboratory, the application herein does not require authentication since the lines are used as standards, not as biological models. Cell lines were selected to represent a range of EGFR expression and different mutation status. H1648, A549, H1355 were routinely cultured in Dulbecco's modified Eagle medium: Nutrient Mixture F-12 containing 10\% fetal bovine serum and $1 \%$ penicillin-streptomycin (Life Technologies, Grand Island, NY, USA). A431 and MCF7 cells were cultured in Dulbecco's modified Eagle medium containing 10\% fetal bovine serum and $1 \%$ penicillin-streptomycin (Life Technologies). H820, H1993, H441, H2882, HCC193, PC9, H1650, H1975 cells were cultured in RPMI 1640 medium containing $10 \%$ fetal bovine serum and $1 \%$ penicillinstreptomycin (Life Technologies). HT29 and SKBR3 were cultured in McCoys medium containing 10\% fetal bovine serum and $1 \%$ penicillin—streptomycin (Life Technologies).

\section{Tissue microarray}

Tissue specimens were prepared in a tissue microarray (TMA) format as previously described. ${ }^{11}$ Representative tumor areas were obtained from formalin-fixed, paraffin-embedded specimens of the primary tumor, and $0.6 \mathrm{~mm}$ cores from each tumor block were arrayed in a recipient block. A NSCLC array, termed YTMA 332, containing 30 tumor cores from NSCLC patients was constructed from patient cases that had complete surgical resection of primary tumor between 2011 and 2013. Those that had adequate residual tumor from primary site were selected to be cored. YTMA 332 also includes a 2-fold redundancy of 15 cell line cores representing a range of EGFR expression (MCF7, HT29, SKBR3, H441, H1355, H1993, H1648, A549, A431, H820, PC9, HCC193, H1975, H2882, H1650). All tissue was used after approval from the Yale Human Investigation Committee protocol \#9505008219, which approved the patient consent forms or in some cases waiver of consent.

\section{Antibody titration}

To optimize the titer for EGFR D38B1 antibody (Supplementary Table 1), a standardization array (YTMA 332) was stained at five concentrations covering two orders of magnitude in serial sections. The antibody was tested at the dilutions $\quad 1: 50 \quad(0.34 \mathrm{ug} / \mathrm{ml}), \quad 1: 100 \quad(0.17 \mathrm{ug} / \mathrm{ml}), \quad 1: 500$ (0.034 ug/ml), 1:1,000 (0.017 ug/ml) and 1:5,000 (0.0034 ug/ $\mathrm{ml})$. A titration curve was plotted using the average scores of the highest 10\% (representing the signal) and lowest $10 \%$ (representing the noise) of the patient tumor cores and the signal-to-ratio. We defined the objectively optimal antibody titer as the one that had the highest dynamic range of signal with the highest signal-to-noise ratio.

\section{Quantitative immunofluorescence}

Fresh $5 \mu \mathrm{m}$ TMA sections were deparaffinized at $60^{\circ} \mathrm{C}$ for $30 \mathrm{~min}$, incubated in xylene (soaking twice for $20 \mathrm{~min}$ ) and rehydrated with ethanol (twice in $100 \%$ ethanol for $1 \mathrm{~min}$, and then in $70 \%$ ethanol for $1 \mathrm{~min}$ ). Antigen retrieval was performed in a PT module (LabVision) with EDTA buffer (Sigma-Aldrich) $\mathrm{pH} 8.0$ for $20 \mathrm{~min}$ at $97^{\circ} \mathrm{C}$. After blocking of endogenous peroxidase with $30 \%$ hydrogen peroxide in methanol, slides were incubated with a blocking solution containing $0.3 \%$ bovine serum albumin in Tris-buffered saline solution and $0.05 \%$ Tween solution for $30 \mathrm{~min}$ at room temperature. Slides were then incubated overnight with a cocktail of EGFR (D38B1, Cell Signaling Technology) rabbit monoclonal antibody and mouse monoclonal cytokeratin antibody clone AE1/AE3 (M3515, Dako). Next, a mixture of Alexa 546 conjugated goat anti-mouse secondary antibody (Molecular Probes, Eugene, OR, USA) diluted 1:100 in rabbit EnVision reagent (K4003, Dako) was applied to the slides for $60 \mathrm{~min}$ at room temperature. Cyanine 5 (Cy5) directly conjugated to tyramide (FP1117; Perkin Elmer) at a 1:50 dilution for $10 \mathrm{~min}$ was used for target detection and ProLong mounting medium (ProLong Gold; Molecular Probes) with 4,6-diamidino-2-phenyl-indole (DAPI) was used to stain nuclei. Control slides were run for reproducibility alongside each experimental slide staining run. Quantitative measurement of EGFR immunofluorescence was performed using AQUA ${ }^{\circledR}$ method (Genoptix Medical Laboratory), quantifying fluorescent signal within subcellular compartments, as described previously. ${ }^{3}$ A tumor mask was created by binarizing the cytokeratin signal and creating an epithelial compartment. A quantitative immunofluorescence score was calculated by dividing the target pixel intensity by the area of cytokeratin compartment. QIF scores were normalized to the exposure time and bit depth at which the images were captured, allowing scores collected at different exposure times to be comparable. All spots were visually evaluated and cases with staining artifacts or presence of less than $2 \%$ tumor area were systematically excluded. 
a

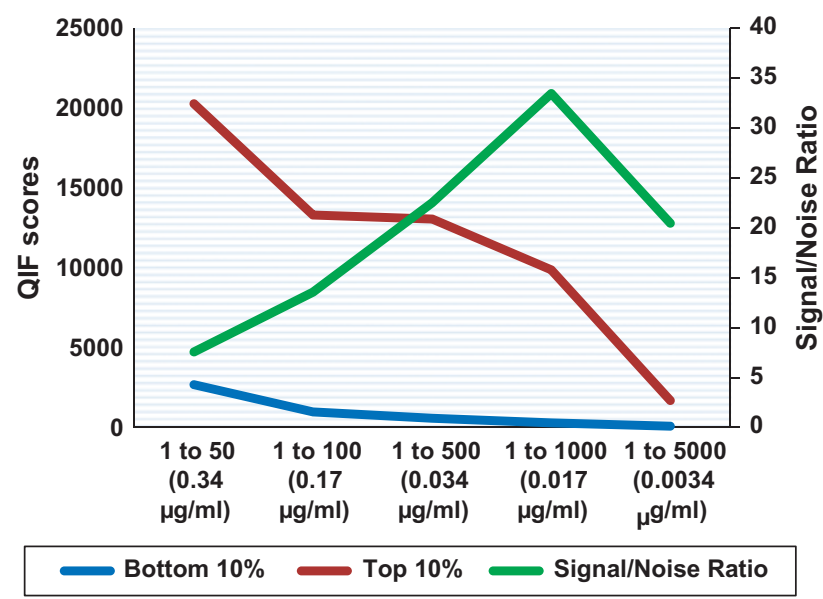

b

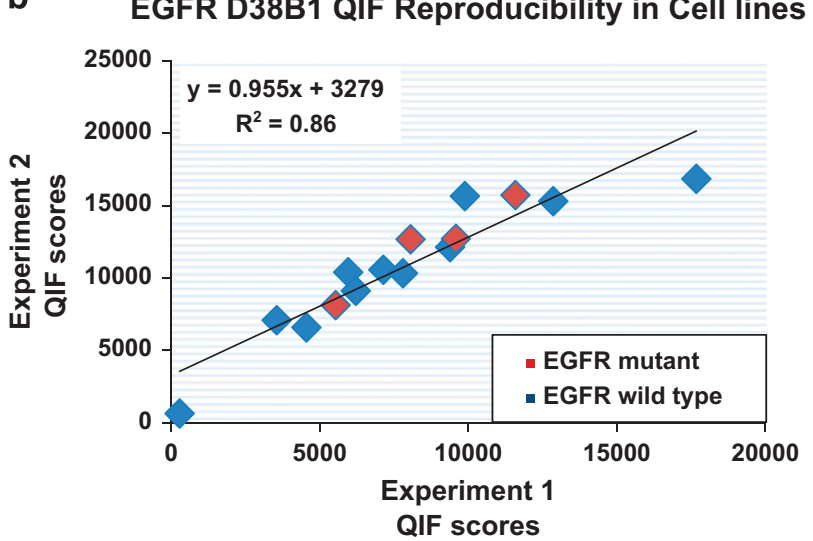

C
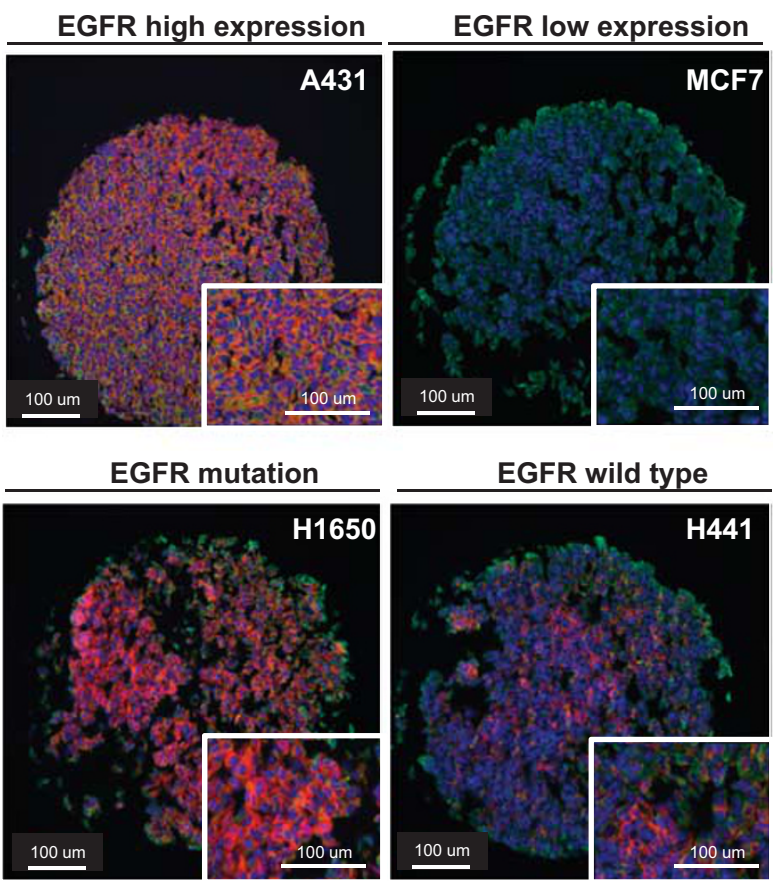

Figure 1 (a) Titration curve of EGFR D38B1 primary antibody plotted at five different concentrations. The blue line shows the average QIF scores of the lowest $10 \%$ patient cases included in the standardization array, representing the noise. The red line shows the average QIF scores of the highest $10 \%$ patient cases included in the standardization array, representing the signal. The green line is the signal-to-noise ratio for each EGFR D38B1 antibody concentration. (b) Reproducibility of EGFR expression quantified by AQUA in the optimal EGFR D38B1 concentration measured by AQUA in 15 cell lines with different EGFR expression. Red dots represent EGFR mutant cell lines and blue dots represent EGFR wild-type cell lines. (c) AQUA images of FFPE cell lines with different EGFR expression level and mutation status. EGFR (red), cytokeratin (green), DAPI (4',6-diamidino-2-phenylindole) (blue). Images are representative of two independent experiments.

\section{Statistical analysis}

Pearson's correlation coefficient (R) was used to assess the correlation between cell lines' QIF scores and the absolute protein concentration measured by MS-LT-SRM. For the statistical analysis, the average marker score obtained from two available cores of each cell line was used.

\section{Quantitative proteomics assay by mass spectrometry- based LT-SRM}

Targeted quantification of EGFR in cell lines by LT-SRM was performed following previously described procedures designed for the quantification of proteomic targets in FFPE tumor tissues. ${ }^{10}$ The steps included (i) preparation of liquid tissue lysates from formalin-fixed cell pellets; (ii) addition of known amount of isotope-labeled internal standard peptides into each cell lysate; and (iii) quantitative analysis using LT-SRM. Detailed methods for above steps, for the peptide separation/elution procedures, mass spectrometry parameters, and for the analysis of the results have been previously described. ${ }^{10}$ The amount of EGFR in each sample (amol per microgram total protein) was calculated from the ratio of analyte to internal standard peak areas, multiplied by the known amount of internal standard spiked and normalized to the total protein amount injected. Samples were analyzed in triplicate.

\section{RESULTS}

The EGFR primary antibody used for QIF was initially titered in a standardization array containing 30 non-small cell lung cancer (NSCLC) patient tumors with a broad range of EGFR expression. To maximize specificity, the primary antibody was tested at five different concentrations covering two orders of magnitude in serial sections, from $0.34 \mathrm{ug} / \mathrm{ml}$ (dilution 1:50) to $0.0034 \mathrm{ug} / \mathrm{ml}$ (dilution 1:5,000), and the signal-to-noise 

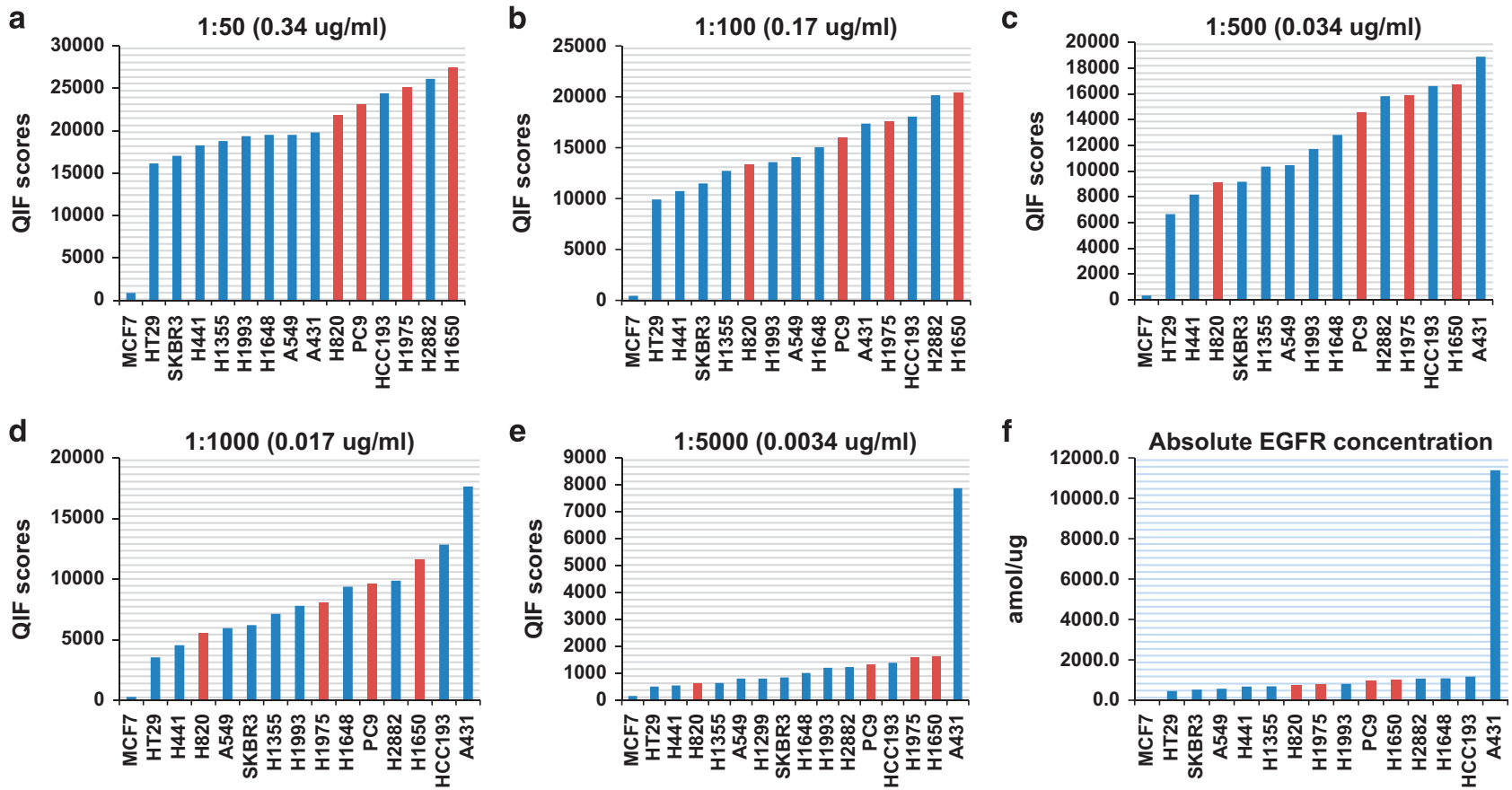

Figure 2 (a-e) QIF scores in the 15 cell lines with different EGFR expression as measured by AQUA at different EGFR D38B1 primary antibody concentrations covering two orders of magnitude in serial sections. (f) Absolute EGFR concentration measured by LT-SRM-MS in 15 cell lines with different EGFR expression. The QIF scoring and the absolute protein concentration by LT-SRM- MS were performed in cores and sections respectively, coming from the same cell pellets. Red bars represent EGFR mutant cell lines and blue bars represent EGFR wild-type cell lines.
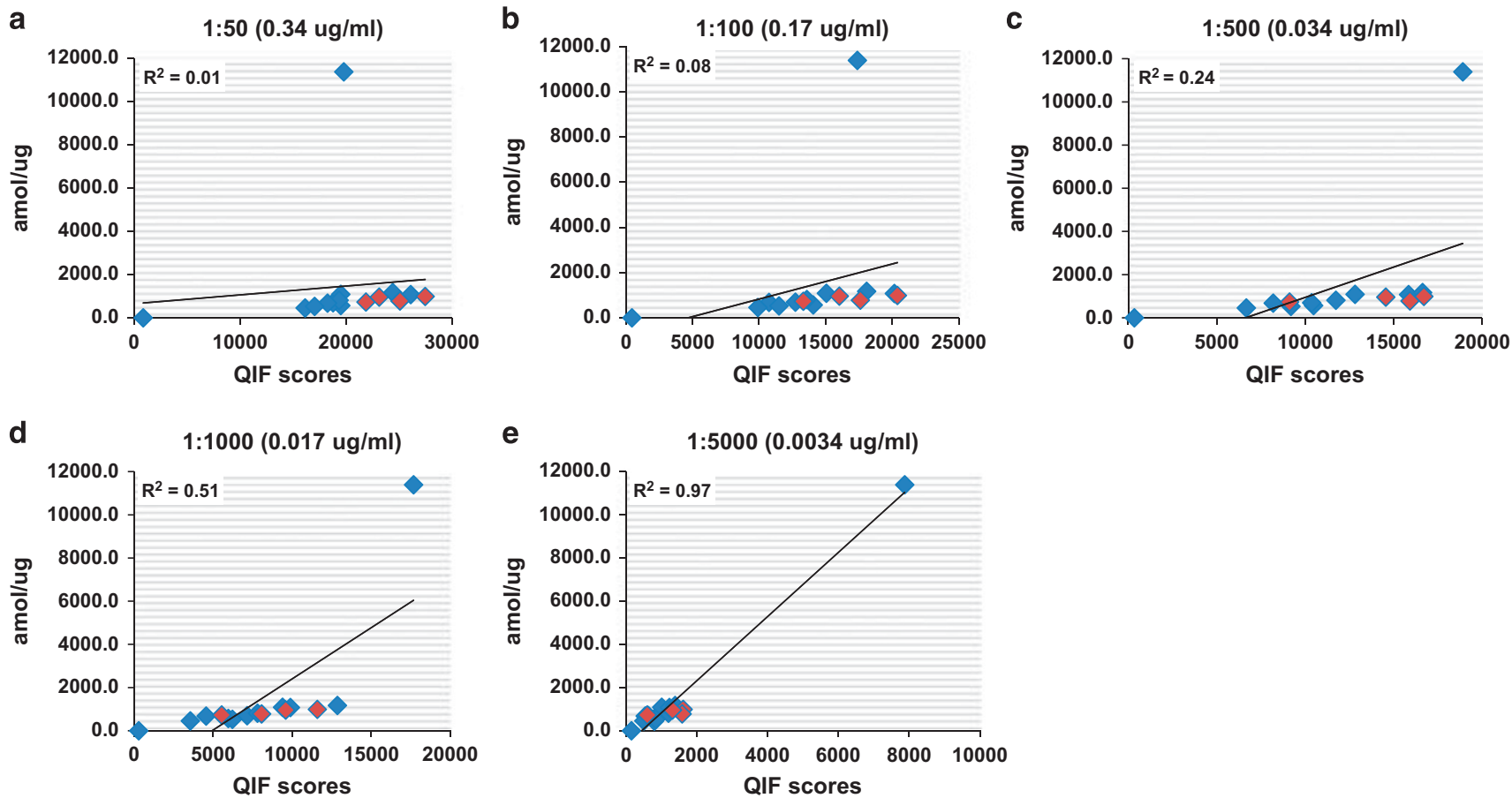

Figure 3 (a-e) Regression charts between absolute EGFR concentration measured by LT-SRM- MS and the QIF scores at different EGFR D38B1 primary antibody concentrations covering two orders of magnitude in 15 cell lines, with A431 included. The QIF scoring and the absolute protein concentration by LT-SRM-MS were performed in cores and sections respectively, coming from the same cell pellets. Red dots represent EGFR mutant cell lines and blue dots represent EGFR wild-type cell lines. 

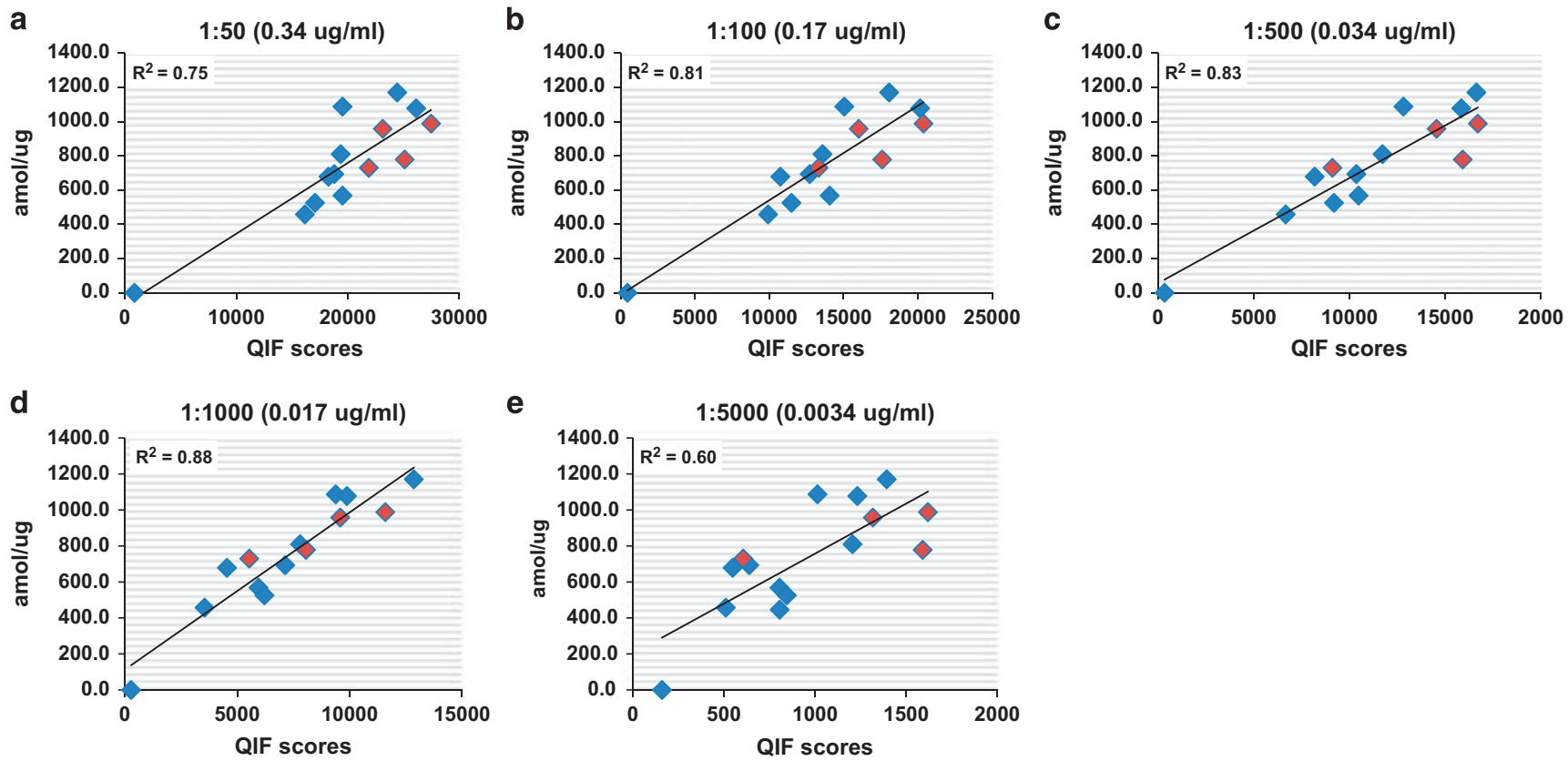

Figure 4 (a-e) Regression charts between absolute EGFR concentration measured by LT-SRM- MS and the QIF scores at different EGFR D38B1 primary antibody concentrations covering two orders of magnitude in 14 cell lines, with A431 not included. The QIF scoring and the absolute protein concentration by LT-SRM-MS were performed in cores and sections respectively, coming from the same cell pellets. Red dots represent EGFR mutant cell lines and blue dots represent EGFR wild-type cell lines.

ratio was calculated by QIF (See Methods). The signal-tonoise ratio was found to be the highest at $0.017 \mathrm{ug} / \mathrm{ml}$ primary antibody concentration (Figure 1a).

In parallel, EGFR expression of 15 cell lines with different EGFR expression levels and mutation status was measured by using the same primary antibody concentrations. EGFR measurement by QIF in the cell lines was highly reproducible when the optimal primary antibody concentration was used, $\mathrm{R}^{2}=0.86$ (Figure $1 \mathrm{~b}$ ). The two assays were stained, imaged and quantified independently. Representative images of the IF staining on different cell lines are shown in Figure 1c and subcellular localization of the IF signal in A431 cell line is shown in Supplementary Figure 1. The QIF scores of the 15 cell lines varied with different EGFR antibody concentration, revealing a wide range of the receptor's expression (Figures 2a-e). The absolute EGFR concentration in attomols (10$18 \mathrm{~mol}$ ) per microgram was measured in the same 15 cell line pellets using the LT-SRM assay (Figure 2f). Mass spectrometry measurement confirmed the wide EGFR expression range but also revealed an almost 10-fold EGFR abundance in A431 compared to the other cell lines.

To compare the agreement of the two assays in target measurement, a regression between the IF scores and the absolute protein concentration measured by LT-SRM was plotted (Figures 3a-e). EGFR expression in A431 exceeded the dynamic range of the IF assay making it the only cell line driving the regression. The high difference of EGFR abundance in this cell line was only shown when the EGFR primary antibody was used at a low concentration, allowing the difference in expression to be distinct (Figure 3e).

When A431 was excluded (Figures 4a-e), allowing the assays to be compared in the same dynamic range, the best agreement between IF scores and LT-SRM absolute protein concentration was found when the EGFR D38B1 primary antibody was used at the optimal signal-to-noise concentration $(0.017 \mathrm{ug} / \mathrm{ml})$, showing a strong linear regression between the two assays $\left(R^{2}=0.88\right)$ (Figure $4 d$ ). The linearity of the agreement decreased when the working concentration moved away from the optimal concentration of the EGFR D38B1 primary antibody. This observation represents an orthogonal method for validation of the concordance between the mass spec and QIF methods.

\section{DISCUSSION}

Validation and optimal titration of the reagents used in antibody-dependent assays is critical. Subtle differences in antibody concentration have been shown to affect the scoring and the signal-to-noise threshold, and even change biomarker associations with outcomes. ${ }^{11,12}$ Here, we have demonstrated that quantitative optimization of titration by calculation of signal-to-noise ratio allows IF to be used to assess absolute protein concentration in a linear and reproducible manner with a validated antibody, in spite of the use of an enzymatic amplification in the detection system. Although this method requires careful validation with every run and the use of antibody-specific tissue microarrays with cases selected to span the dynamic range of expression, this data supports that 
IF can be as reproducible and objective as nucleic acid methods.

This study has a number of limitations. Firstly, all the results come from cell line studies and the absolute protein quantification potential of QIF on patient derived tumors has not been validated yet. Secondly, both QIF and MS were performed on FFPE patient tissue and cell lines processed with the standard histopathological methods used in the clinical setting. The use of IF in other preparations (frozen tissue, etc) would need to be validated separately. Also, this study does not address the issue of pre-analytic variables and loss of epitope availability as function tissue damage before stabilization. By depending heavily on cell lines, we control many pre-analytic variables that may affect tissue. There are not yet methods to normalize for pre-analytic tissue damage, in the way 'housekeeping' genes are used to normalize some nucleic acid-based tests. However, the use of core biopsies followed by immediate fixation represents a method to minimize tissue damage and is also a popular substrate for companion diagnostic tests. Regardless of the method chosen for tissue acquisition, we argue that QIF can accurately measure the epitope present on the slide, with or without the effects of pre-analytic variables.

It is often impossible to determine the cause for the failure of biomarker-driven clinical trials, but the absence of accurate quantitative tests may be one explanation. Currently, protein expression in clinical tissue samples is typically assessed by IHC. IHC is often used in a manner that is subjective with limited reproducibility. Here we show that QIF can be standardized to MS to allow quantitative (accurate, linear, and reproducible) measurement of a biomarker protein (EGFR) on glass slides. We hope this work debunks the common perception that IHC or IF can never be quantitative and opens the door to better quality, more accurate, protein-based companion diagnostic testing.

\section{Supplementary Information accompanies the paper on the Laboratory} Investigation website (http://www.laboratoryinvestigation.org)

\section{ACKNOWLEDGMENTS}

This work was supported by grants from the NIH including the Yale SPORE in Lung Cancer, P50-CA196530, the Yale Cancer Center Support Grant, P30-CA016359, support from the OKIBEE support grant (KS) and support from Nantomics and Gilead Sciences (DR).

\section{DISCLOSURE/CONFLICT OF INTEREST}

In the past 24 months DLR has been engaged in the following relationships: as a consultant to Astra Zeneca, Agendia, Bethyl Labs, Biocept, BMS, Cell Signaling Technology, Cernostics, ClearSight, FivePrime, Genoptix/Novartis, Metamark Genetics, Merck, OptraScan, Perkin Elmer, and Ultivue. He has received honoraria for presentations at Genentech/Roche, Cell Signaling Technology, and Ventana. He holds equity in Metamark Genetics. Cepheid, Genoptix, Gilead Sciences, Pierre Fabre, Perkin Elmer and Nantomics fund research in his laboratory. FC and $\mathrm{TH}$ are employees of Nantomics Inc. The remaining authors declare no conflict of interest.

1. Hsi ED. A practical approach for evaluating new antibodies in the clinical immunohistochemistry laboratory. Arch Pathol Lab Med 2001;125:289-294.

2. Allred DC, Harvey JM, Berardo M, Clark GM. Prognostic and predictive factors in breast cancer by immunohistochemical analysis. Mod Pathol 1998;11:155-168.

3. Camp RL, Chung GG, Rimm DL. Automated subcellular localization and quantification of protein expression in tissue microarrays. Nat Med 2002;8:1323-1327.

4. Aebersold R, Mann M. Mass spectrometry-based proteomics. Nature 2003;422:198-207.

5. Ong SE, Mann M. Mass spectrometry-based proteomics turns quantitative. Nat Chem Biol 2005;1:252-262.

6. Addona TA, Abbatiello SE, Schilling B, et al. Multi-site assessment of the precision and reproducibility of multiple reaction monitoring-based measurements of proteins in plasma. Nat Biotechnol 2009;27:633-641.

7. Nilsson T, Mann M, Aebersold R, Yates 3rd JR, Bairoch A, Bergeron JJ. Mass spectrometry in high-throughput proteomics: ready for the big time. Nat Methods 2010;7:681-685.

8. Prieto DA, Hood BL, Darfler MM et al. Liquid tissue: proteomic profiling of formalin-fixed tissues. Biotechniques 2005;38(S6):S32-S35.

9. Nishimura $T$, Nomura $M$, Tojo $H$, et al. Proteomic analysis of lasermicrodissected paraffin-embedded tissues: (2) MRM assay for stagerelated proteins upon non-metastatic lung adenocarcinoma. J Proteomics 2010;73:1100-1110.

10. Hembrough T, Thyparambil S, Liao $\mathrm{WL}$, et al. Selected reaction monitoring (SRM) analysis of epidermal growth factor receptor (EGFR) in formalin fixed tumor tissue. Clin Proteomics 2012;9:5.

11. McCabe A, Dolled-Filhart M, Camp RL, Rimm DL. Automated quantitative analysis (AQUA) of in situ protein expression, antibody concentration, and prognosis. J Natl Cancer Inst 2005;97:1808-1815.

12. Welsh AW, Moeder CB, Kumar S, et al. Standardization of estrogen receptor measurement in breast cancer suggests false-negative results are a function of threshold intensity rather than percentage of positive cells. J Clin Oncol 2011;29:2978-2984. 